gleich, so ist der Nenner ein Maximum. Im Allgemeinen gilt daber die Formel:

$$
s>\frac{[\text { val. abs. } \lambda]}{l} \frac{\lambda \text {. }}{\mathrm{n} \mathbf{r}} \text {. }
$$

§. 8. Uebersichtliche Beurtheilung des Gradeg der Annkherung von Formel (1). Die strengen Formeln (24) und (39) sind so complicirt, dass man sicher lieber statt $\mathcal{S}$ den mittleren Fehler $\mu$ berechnen wird. Wollte man nun doch (1) benutzen, so gilt die Regel:

Die Annäherung der Formel (1) ist un so grösser, je weniger nach der erfolgten Ausgleichung die Gewichteder verbesserten Beobachtungenvon einander abweichen. Eingangs haben wir die gegebenen Beobachtungen auf gleiches Gewieht reducirt gedacht. Sei nun z. B. bei bedingten Beobachtungen $\mu$ der mittlere Fehler einer (reducirten) Beobachtung, so ist (vergl. Seite 178 und 179 meiner Ausgleichungs-Rechnung) das Quadrat des mittleren Fehlers einer verbesserten Beobachtung $\left(l_{k}+\lambda_{k}\right)$ gleich

(41) $\mu_{k}^{2}=\mu^{2}\left(1-\left(p_{k}^{2} Q_{1.1}+2 p_{k} q_{k} Q_{1.2}+q_{k}^{2} Q_{2 \cdot 2}\right)\right)$. Ein Blick auf (39) zeigt die Strenge unserer Regel Nunmebr sieht man auch deutlich, dass bei directen Beobachtungen Formel (1) streng gelten muss, aber bei vermittelnden und bedingten Beobachtungen nicht gelten wird.
§ 9. Ein gcheinbares Paradoron. Nach Formel (8) ist für 2 directe Beobachtungen

$$
s=\frac{d}{V / \overline{2}}
$$

bekanntlich ist aber für den mittleren Fehler $\mu$ alsdann

$$
\mu^{2}=\frac{d^{2}}{2} ; \mu=\frac{d}{12} \text {. }
$$

Dics giebt merkwiirdig genug $\mu=9$, während eigentlich $\mu=1,259$ ist (unendlich viele Beobachtungen vorausgeset $\%$ t). Man muss aber bedenken, dass diese Formeln erst genaue Resultate geben, wenn man für $d$ resp. $d^{2}$ einen mittleren Werth aus $n_{1}$ Beobachtungspaaren einsetzt, wo $n_{1}$ eine grosse Zabl ist:

$$
\vartheta=\frac{[\mathrm{d}]}{\mathrm{n}_{1} V \sqrt{2}} ; \mu^{2}=\frac{\left[\mathrm{d}^{2}\right]}{\mathrm{n}_{1} \cdot 2}
$$

und nun sieht man sogleich, dass 9 und $\mu$ nicht melir gleich sein werden. Nur bei der fehlerhaften Bestimmung von $\mu$ mittelst des Durchschnitts der ersten Potenzen der $\mathrm{d}$ wïrde $\mu$ immer gleich 9 erhalten werden.

Hierin liegt ein drastisches Beispiel dafïir, wie nothwendig es ist, immer bei Vereinigung mebrerer Bestimmungen von $\mu$ auf die Quadrate der $\mu$ zu recarriren.

\begin{tabular}{|c|c|c|c|c|}
\hline 1875 & - & $\begin{array}{l}\text { Mittl. } \\
\text { Mosk. Zeit }\end{array}$ & $\alpha \not \zeta-\alpha *$ & Scheinb. $\alpha \mathscr{b}$ \\
\hline März 24 & 1 & $8^{\mathrm{h}} 13^{\mathrm{m}} 54^{\mathrm{a}} .6$ & - 29.96 & $1^{\mathrm{t}} 32^{\mathrm{m}} 25^{\prime} .32$ \\
\hline 25 & $\mathbf{m}$ & $\begin{array}{lll}8 & 6 & 35.4\end{array}$ & $+3^{m} 21.21$ & $135 \quad 51.52$ \\
\hline 27 & $\mathbf{n}$ & $\begin{array}{lll}7 & 49 & 5.5\end{array}$ & +118.32 & 14252.24 \\
\hline 30 & p & $\begin{array}{llll}8 & 10 & 57.1\end{array}$ & +235.23 & 15347.23 \\
\hline April 5 & $q$ & $\begin{array}{lll}8 & 5 & 25.9\end{array}$ & -841.16 & $\begin{array}{lll}2 & 15 & 16.51\end{array}$ \\
\hline 10 & 8 & $\begin{array}{lll}8 & 10 & 42.0\end{array}$ & $-\quad 4.33$ & 22840.95 \\
\hline
\end{tabular}

Aachen, den 31. März 1875.

\title{
Beobachtungen des Encke'schen Cometen auf der Moskauer Sternwarte,
} gemacht vou Prof. Bredichin.

Mittlere Oerter der Vergleichsterne ftir 1875.0 .
$1=$ B. A. C. 500

$\mathrm{m}=$ B. A. C. 495

$1^{\text {th }} 32^{\mathrm{m}} 56^{\circ} .29$

13231.32

14134.92

15113.00

$2 \quad 2358.59$

$q=$ B. A. C. 771

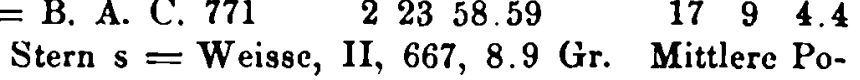
sition für 1875.0 angenommen:

$$
\alpha=2^{b} \cdot 28^{\curvearrowleft} 46^{\bullet} .19
$$

Zahl Mittl.

d.Vgl. Mosk. Zeit $\delta \& 4-\delta *$

$48^{\circ} 7^{m} 16^{\prime} .5+5^{\prime} 46^{\prime \prime} 2$

$\begin{array}{lllll}2 & 74916.2+448.4\end{array}$

$3 \quad 74717.3+3 \quad 37.6$

$474813.4+42.7$

$174630.6+350.0$

$\begin{array}{llllll}8 & 0 & 32.9 & +1 & 13.4\end{array}$

Scheinb. $\delta \&$ d.Val $+15^{\circ} 52196$

$\begin{array}{llll}16 & 4 & 16.5 & 2\end{array}$

$\begin{array}{llll}16 & 27 & 31.2 & 4\end{array}$

$\begin{array}{llll}16 & 56 & 30.2 & 2\end{array}$

$\begin{array}{llll}17 & 12 & 56.4 \quad 2\end{array}$

$\begin{array}{lll}16 & 5 & 5.1\end{array}$ $\delta=+163^{\circ} 49^{\prime \prime} 6$.
Die Vergleichungen der Beobachtungen mit der
155928.6

$1623 \quad 53.6$

165548.0

$\begin{array}{ccc}17 & 9 & 4.4 \\ \text { Mittlere } & \text { Po- }\end{array}$ Epbemeride des $\boldsymbol{H}$. von Astm geben folgende Zahlen: Beob. - Ephem.

\begin{tabular}{rccc} 
& \multicolumn{1}{c}{$\Delta \alpha$} & \multicolumn{1}{c}{$\Delta \delta$} \\
Mär\% 24 & +2.32 & $+\quad 0^{\prime \prime 3}$ \\
25 & +2.60 & -5.4 \\
27 & +2.66 & -11.5 \\
30 & +1.36 & -26.3
\end{tabular}




$$
\begin{array}{rcc} 
& \multicolumn{2}{c}{\text { Beob. }- \text { Ephem. }} \\
\text { April } 5 & \triangle \alpha & \triangle \delta \\
10 & -1.64 & -2^{\prime} 34^{\prime \prime} 9 \\
& -12.4 & -3^{\prime} 11^{\prime \prime} 4
\end{array}
$$

Ausser diesen Beobachtungen babe ich noch 11 Beobachtungen des Encke'schen Cometen angestellt
(Februar 25, 27, 28; März 1, 2, 5, 9, 10, 16, 23 und 28), die werden von mir bald reducirt und den Astr. Nachr. mitgetbeilt werden.

Moskauer Sternwarte, 1875, April 9.

Prof. Th. Bredichin.

\section{Schreiben des Herrn Tisserand, Directors der Sternwarte in Toulouse, an den Herausgeber.}

Le 28 Arril dernier, à 14 heures temps moyen de Toulouse, M. Perrotin observait une planète de 11 ème grandeur, avec les coordounécs

$$
\begin{aligned}
& \text { AR. }=15^{n} 8^{\mathrm{m}} 46 \\
& \delta=-15^{\circ} 39^{\prime} 5
\end{aligned}
$$

La planète la plus voisine, du Berliner Jahrbuch ètait (110) Lydia, dont les coordonnées, déduites seulement do l'éphéméride annuelle, étaient approximativement

$$
\mathrm{AR} .=15 \mathrm{b5} \mathrm{m} \quad \delta=-1707^{\prime}
$$

cause de la grande différence en ascension droite et déclinaison, nous pensions avoir rencontrée une nouvelle planète.

Mais, en partant des éléments elliptiques de (110), contenus dans le Berliner Jahrbuch de 1873 , j'ai trouvé qu'au moment de l'observation, on avait pour (110)

$$
\mathrm{AR} .=15^{\mathrm{b}} 6^{\mathrm{m}} 8^{\prime} \quad \delta=-15^{\circ} 23^{\prime} 1
$$

il en resulte dunc que la planète suppséeo nouvelle est la planète Lydia.

Toulouse, le 1. Mai 1875.

\section{P. Tisserand.}

\section{Elemente des Planeten (139).}

The following element of Asteroid (139) were computed from Professor Walson's observation at Peking (Astr. Nachr. Nr. 2020). The method employed was that of adjusting a circular orbit by variation of the geocentric distances. In the comparison all of the published observations from Oct. $10^{\text {th }}$ to $17^{\text {th }}$ in lusive were used except that of the $16^{\text {th }}$.

Epoch 1874, Oct. 14.5, Greenwich uiean time

M $268047^{\prime} 32^{\prime \prime} 1$

$\left.\begin{array}{llll}\pi & 115 & 31 & 56.7 \\ 8 & 358 & 37 & 28.9\end{array}\right\}$ Ecliptic \& Meau Eq. 1874.0

$\begin{array}{lllll}\text { i } & 8 & 19 & 18.1\end{array}$

甲 25631.6

$\log 20.44933$ $\log \mu 2.876011$

$\mu 751^{\prime \prime} 642$.

$\begin{array}{ccc}\text { Residuals } & - \text { (Obs. } & - \text { Comp.) } \\ \text { Date } & \triangle \lambda & \Delta \beta \\ \text { Oct. } 10 & -1^{\prime \prime} 1 & -2{ }^{\prime \prime} 8 \\ 11 & +0.1 & +0.0 \\ 13 & -4.5 & -2.3 \\ 13 & -2.0 & -(14.3) \\ 13 & +3.1 & -4.6 \\ 14 & -1.7 & -1.6 \\ 15 & +4.8 & +(8.1) \\ 17 & -0.6 & -0.1\end{array}$

Washington, March 26, 1875.

C. L. Doolittle. O. S. Wilson.

In halt:

Za Nr. 2038-2039. E. von Asten. Ueber Encke's Conet. 347. - Helmert. Ueber die Forneln für den Durchachnittsfehler. 363. Bredichill. Beobachtungen des Encke'schen Cometen aul der Moskauer Sternwarte. 365. - P. Tisserand. Sehreiben an den Herausgeber. 367. - C. L. Doulitlle und O. S. Wilsnn. Elemente des Planelen (139). 